\title{
A Survey on Analysis of a Noisy Image by using External and Internal Correlations
}

\author{
Pratima \\ Scholar \\ Department of Computer \\ Science \& Engg \\ BBAU Lucknow
}

\author{
Jitendra Kurmi \\ Assistant Professor \\ Department of Computer \\ Science \& Engg \\ BBAU Lucknow
}

\begin{abstract}
When a noisy image is a single image then it suffers from limited data collection in denoising it. In this paper, we propose image denoising scheme, which explores both internal and external correlation with the help of web images. In this paper, we use two stage filtering technique for denoising the image in the first stage we use graph-cut based patch matching and frequency truncation and then combining result of both filter and enter into the second stage in the second stage we use adaptive filtering and wiener filtering for denoising the noisy image and then combining the result of both filter. By using two stage filtering technique we get better filtered image.
\end{abstract}

\section{Keywords}

Image denoising, external correlations , Internal correlations, web images, wiener filter, adaptive filter.

\section{INTRODUCTION}

Digital image plays an important role in our daily life and in the area of research and technology. When the digital image is transmitted from one place to another place, during the transmission noise is added into the image. Any form of signal processing having image as an input and output is called image processing. Image processing is a method to perform some operations on an image, in order to get an enhanced image or to extract some useful information from it. It is a type of signal processing in which input is an image and output may be image or characteristics/features associated with that image. There are two types of methods used for image processing namely, analogue and digital image processing. The purpose of image processing is divided into 5 groups. They are Visualization, Image sharpening and restoration, Image retrieval, Measurement of pattern, Image Recognition.

Digital images are often degraded by noise in the acquisition and transmission phase. The goal of image de-noising is to recover the true original image from such a distorted noisy copy. The presence of noise not only produces undesirable visual quality but also lowers the visibility of low contrast objects. The image usually has noise which is not easily eliminated in image processing. According to actual image characteristic, noise statistical property and frequency spectrum distribution rule, people have developed many methods of eliminating noises, which approximately are divided into space and transformation fields. The space field is data operation carried on the original image, and processes the image grey value, like neighborhood average method, wiener filter, center value filter and so on. Various types of noise present in image are Gaussian noise, Salt \& Pepper noise and Speckle noise. Image de-noising techniques are used to prevent these types of noises while retaining the important signal features. Poor image sensors, imperfect instruments, problems with data acquisition process, transmission errors and interfering natural phenomena are its main sources. Therefore, it is necessary to detect and remove noises present in the images. Reserving the details of an image and removing the random noise as far as possible is the goal of image de-noising approaches.

Image denoising is defined as the problem of recovering a natural image I from its noise-corrupted image IN. This problem has a rich history, with considerable progress made in recent years. In particular, the idea of using recurrence of small image patches within a natural image for denoising was first introduced in [4], and later extended by [10]). In these methods, each noisy image patch is denoised using other noisy patches within the noisy image. We refer to these as "Internal Denoising" methods. Other recent patch-based denoising methods employ external clean natural image patches (or a compact representation of them) to denoise each patch (e.g., [5]). We refer to these as "External Denoising" methods. Internal and External denoising were previously compared by [13] in the context of the 'Non-Local Means' (NLM) settings [4]. They found Internal NLM to be superior to External NLM. However, their comparison assumed that the same method (Internal or External) was applied to all image patches. Applying the same method to all image patches is typically true for most denoising methods. In this paper we show that some image patches inherently prefer Internal Denoising, whereas other patches inherently prefer External Denoising. Combining the best of both should thus lead to better results. We analyze and quantify the Internal vs. External preference of small noisy patches pn , and show that it is tightly related to the 'Signal-to-Noise-Ratio' within the patch, denoted by PatchSNR(pn). This is the ratio between the empirical variance of the original clean patch $p$, and the empirical variance of the noise within its noisy patch pn. We show that patches with low PatchSNR (e.g., in smooth image regions) tend to prefer Internal denoising, whereas patches with high PatchSNR (e.g., edges, texture) tend to prefer External denoising. The reason for this dichotomic behavior is the tradeoff between noise-fitting vs. signal-fitting. Patches with low PatchSNR are dominated by the noise. Unconstrained External search tends to overfit their noise (as opposed to constrained Internal denoising). On the other hand, patches with high PatchSNR (e.g., edges, texture) are dominated by the signal. They are less prone to overfitting the noise in an unconstrained External search, yet, they have much better signal-fit in the external clean database. These tradeoffs are analyzed and quantified. Finally, we exemplify the power of the PatchSNR, by combining pairs of different Internal/External denoising methods using a simple threshold 
on the patchSNR value (estimated per patch from the noisy image). Such a simple combination provides results better than the current state of- the-art methods.

\section{- Internal Denoising}

Single image based (internal) denoising methods aim to recover image details by exploring the correlations inside the noisy image itself. Earlier approaches focus on pixel-level denoising methods, which only explore the correlations between a noisy pixel and its neighbouring pixels, such as Gaussian filtering, bilateral filtering, anisotropy diffusion, or total-variation regularization. Hereafter, exploring the correlations between non-local patches has boosted denoising performance, such as non-local means[4], BM3D, low-rank regularization, learned simultaneous sparse coding (LSSC), and high order singular value decomposition. These methods group similar patches in the noisy image and then recover their common structures by spacial domain averaging, transform domain filtering, jointly sparsity constrain in the learned dictionary domain, or by using singular value decomposition. These methods work well for images containing repetitive patterns. However, these methods cannot well handle high noise levels since the patch structures are heavily polluted by the noise. Therefore, the multi-scale strategy is introduced in denoising when the noise level is high. Since the noise is uncorrelated across neighbouring pixels, down-sampling will reduce the noise level and make image structures more visible. In addition to simple downsampling, there is also a multi-scale sparse representation based denoising algorithm. Furthermore, M. Zontak et al. propose using patch recurrence across scales to recover the signal. The above mentioned denoising methods tend to smooth texture regions. Therefore, a texture enhanced method by enforcing the gradient distribution of the denoised image to be close to the estimated gradient distribution is proposed. However it tends to produce much lower PSNR values compared with state-of-the-art methods since the estimated gradient distribution may deviate from the ground truth. Unlike these methods, we propose using external correlated images to help improve the visual quality of texture regions, while improving objective qualities.

\section{- $\quad$ External Denoising}

Learning based (external) denoising methods which benefit from certain priors learned from noise free images (or the noisy image itself) have recently become quite numerous. KSVD-based training has extended from the noisy image itself to 10000 natural images to learn the bases. Statistical models learned from a large scale of natural image patches have shown its feasibility in image denoising, such as highorder MRF models and the finite Gaussian mixture model [5](trained over 2 million natural image patches). In addition, the deep learning method has shown its power in image denoising. In the relationship between a noisy patch and its noise free version has been studied by a plain multi-layer perceptron deduced from a large training database $(150000$ images). All these works have demonstrated the effectiveness of using natural image priors to help remove noise. However, since they use general priors for all kinds of noisy images, without considering the content of the noisy image, they soon reach their performance limitation (comparable to BM3D) and tend to introduce artifacts if the noisy image doesn't satisfy the assumed priors. Different from them, we propose adaptively changing the external dataset for each noisy image by using content based image retrieval technologies.

\section{- Internal And External Combined Denoising}

The single image based (internal) and learning based (external) denoising methods have complementary strengths. Therefore, they can be combined to improve denoising performance. I. Mosseri et al. propose classifying the patches into internal and external denoising according to patch's signal-noise-ratio (SNR)[6].Previous work [7] presents a simple and effective frequency domain fusion method to combine the advantages of internal and external denoising results and achieves the best denoising result compared with stand-alone methods.

\section{RELATED WORK}

Yipin Zhou "Explore the Power of External Data in Denoising Task",et al.(2006) [6] - According to the author the goal of this paper is to explore the power of external data in the image denoising task, that is, to show that with taking advantage of an immense amount of information provided by external datasets, external denoising method should be more promising than internal denoising method which only extracts information from the input noisy image itself. In this paper, the author present a simple external denoising method which combines Non Local Means (NLM) with a randomized patch matching algorithm to denoise the input image (with an large enough external dataset) efficiently. Experimental results on a large set of images demonstrate that this external denoising method can outperform the according internal NLM and be competitive with the method which properly combine the denoising results of both internal and external NLM. This experiment demonstrate that applying external data in denoising task is more promising and powerful than internal data, though the patch noise overfitting problem is more severe for external approach. To alleviate this problem, the author propose an extension applying adaptive patch size.

Kostadin Dabov "Image denoising by sparse 3D transform-domain collaborative filtering",et al.( 2007) [10]- The author propose a novel image denoising strategy based on an enhanced sparse representation in transform domain. The enhancement of the sparsity is achieved by grouping similar 2D image fragments (e.g. blocks) into 3D data arrays. Collaborative filtering is a special procedure developed to deal with these $3 \mathrm{D}$ groups. There are three successive steps used:3D transformation of a group, shrinkage of the transform spectrum, and inverse 3D transformation. The result is a $3 \mathrm{D}$ estimate that consists of the jointly filtered grouped image blocks. A significant improvement is obtained by a specially developed collaborative Wiener filtering. The proposed approach can be adapted to various noise models such as additive colored noise, non-Gaussian noise, etc., by modifying the calculation of coefficients' variances in the basic and Wiener parts of the algorithm. In addition, the developed method can be modified for denoising 1D-signals and video, for image restoration, as well as for other problems that can benefit from highly sparse signal representations.

Harold Christopher Burger "Improving Denoising Algorithms via a Multi-scale Meta-procedure",et al.(2011) [11] - The author explains that many state-of-the-art denoising algorithms focus on recovering high-frequency details in noisy images. However, images corrupted by large amounts of noise are also degraded in the lower frequencies. Thus properly handling all frequency bands allows for better denoise in such regimes. To improve existing denoising algorithms the author propose a meta-procedure that applies existing denoising algorithms across different scales and combines the resulting images into a single denoised image. The comprehensive experiments shows that several state-of- 
the-art image denoising algorithms can be improved using this approach. Even though BM3D is arguably one of the best currently existing denoising algorithms, this method was able to improve its results on images that have been corrupted by high noise levels.

Maria Zontak "Separating Signal from Noise using Patch Recurrence Across Scales", et al.(2013) [12]- This paper shows how the multi-scale property can be extended to solve ill-posed problems under noisy conditions, such as image denoising. While clean patches are obscured by severe noise in the original scale of a noisy image, noise levels drop dramatically at coarser image scales. This allows for the unknown hidden clean patches to "naturally emerge" in some coarser scale of the noisy image. The author present a strong multi-scale prior for solving ill posed problems under severe noise: he observe that almost every noisy image patch has a clean version of itself hiding in some coarser scale of the image, at the same relative image coordinates. This restrictive search space forms a strong prior for separating the signal from the noise. Incorporating this prior into a simple denoising algorithm yields state-of-the-art denoising results. Combining this prior with more sophisticated denoising methods is likely to lead to further improvement. Finally, beyond denoising, he believe this can serve as a strong prior for other ill-posed problems, like edge-detection in noisy images, super-resolution in presence of noise, and more.

Huanjing Yue "CID: Combined Image Denoising in Spatial and Frequency Domains Using Web Images",et al.(2014) [7] - The author propose a novel two-step scheme to filter heavy noise from images with the assistance of retrieved Web images. First, for every noisy image block, he build two three dimensional (3D) data cubes by using similar blocks in retrieved Web images and similar nonlocal blocks within the noisy image, respectively. To better use their correlations, he propose different denoising strategies. The denoising in the 3D cube built upon the retrieved images is performed as median filtering in the spatial domain, whereas the denoising in the other $3 \mathrm{D}$ cube is performed in the frequency domain. These two denoising results are then combined in the frequency domain to produce a denoising image. Second, to handle heavy noise, we further propose using the denoising image to improve image registration of the retrieved Web images, 3D cube building, and the estimation of filtering parameters in the frequency domain. Afterwards, the proposed denoising is performed on the noisy image again to generate the final denoising result. Experimental results showed that their scheme significantly outperforms the four state-of-the-art methods in both subjective and objective evaluations. He would like to point out that the correlated Web images play an important role in our CID. Without any correlated images, CID degenerates into one frequency filtering in the intrinsic cube and provides a similar performance as BM3D.

\section{SYSTEM MODEL}

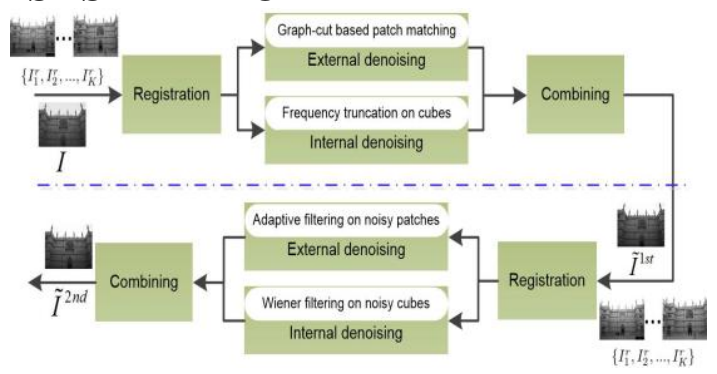

i. Correlated Image Retrieval And Registration

a) Correlated Image Retrieval - Previous learning based denoising methods ignore content priors in a noisy image, which limits improvement in denoising performance. Therefore, we adopt content-based image retrieval technology, specifically the scale invariant feature transform (SIFT) based method proposed in [8],to retrieve correlated images from a large-scale database, as our external dataset. Since a large scale SIFT feature may cover multiple small scale SIFT features, therefore L. Dai et al. propose bundling one large scale SIFT with many small scale SIFT features, namely using a visual group as one retrieval unit [8]. The visual group is much more robust than the quantized single SIFT feature because the relative positions of SIFT features are considered in matching. After matching all the visual groups extracted from the noisy image with those extracted from candidate images, we obtain a set of correlated images.

b) Correlated Image Registration - The correlated images, though similar, are usually taken in different viewpoints, focal lengths and illuminations. Searching for matched patches from these images directly will not only impose considerable computational burdens, but also decrease the matching accuracy since the best matched patch may be at a different rotation and scale of the candidate patch. Although the patch matching algorithm proposed in [9] could search for patches across scales and rotations, it will result in an incorrect result because of the optimization process and the missing of true signal information with noisy query. To solve this problem, we propose an approximate alignment through geometric registration to improve the correlation between the noisy query and retrieved images.

ii. Combined Image Denoising: The First Stage

c) Denoising by Exploring External Correlations using Baseline Method - An intuitive method is to use $l_{2}$ distance as the matching criterion. Let $\mathbf{t}_{i}=\left(x_{i}, y_{i}, z_{i}\right)$ be the translation vector (in col, row, and image number directions) of the $i^{t h}$ patch from the noisy image to the reference images. We want to find the optimal translation for each patch by minimizing the following function:

$$
E(\mathrm{t})=\sum D\left(\mathrm{P}_{i}, \mathrm{Q}\left(\mathrm{t}_{i}\right)\right) i
$$

where $D\left(\mathbf{P}_{i}, \mathbf{Q}\left(\mathbf{t}_{i}\right)\right)$ is ${ }_{2}$ distance of patch $\mathbf{P}_{i}$ and the candidate patch $\mathbf{Q}$ with translation $\mathbf{t}_{i}$. Note that the mean values of all the candidate patches and the noisy query have been removed to reduce illumination effect.

The translation vectors are obtained by minimizing the following energy function.

$$
\begin{gathered}
E(\mathrm{t})=\sum D\left(\mathrm{P}_{i}, \mathrm{Q}\left(\mathrm{t}_{i}\right)\right)+\beta \sum S\left(\mathrm{t}_{i}, \mathrm{t}_{j}\right) \\
i \quad(i, j) \in N
\end{gathered}
$$

where $D\left(\mathbf{P}_{i}, \mathbf{Q}\left(\mathbf{t}_{i}\right)\right)$ is the same as that defined in $S\left(\mathbf{t}_{i} \mathbf{t}_{j}\right)$ is the smoothness term, $\beta$ is a weighting parameter to adjust the effect of the smooth constrain and $(i, j) \in N$ denotes that the $i t h$ and $j$ th patch are neighboring patches. In this paper, $\mathrm{N}$ is a four connected grid. The smooth term $\mathrm{S}$ penalizes the differences of neighboring translation vectors.

\section{a) Denoising by Exploring Internal Correlations}

The internal correlations are explained in a way that is similar to BM3D.For each noisy patch $\mathrm{P}$, we search its $\mathrm{k}$ nearest neighbour $(k-\mathrm{NN})$ patches from $I$ with $\ell_{2}$ distance as 
matching criteria, building a 3D cube $\mathrm{P}_{3 D}$. Then we apply a 3D transform and filter the noise by hard thresholding in the transform domain. It is described as follows:

$$
\mathrm{P}_{3 D}=T^{-1}\left(H\left(T_{3 D}\left(\mathrm{P}_{3 D}\right), \lambda_{3 D} \sigma\right)\right)
$$

$3 D$

where $T_{3 D}$ represents the $3 \mathrm{D}$ transform consisting of a $2 \mathrm{D}$ wavelet transform and a 1D Hadamard transform along the third dimension, and $\mathrm{H}($.) represent the hard thresholding operator of the transform coefficients with the given threshold $\lambda_{3 D} \sigma$. After the inverse 3D transform $T^{-1}$, we obtain the denoise $3 D$ intrinsic cube P3D. We use Pi to represent the internal denoising result of the noisy patch $\mathrm{P}$.

\section{b) Combined internal and external denoising result}

The internal denoising method is good at recovering lowfrequency information, since similar patches are from the same noisy image and have similar structures and illumination However its high-frequency information is filtered out by the hard thresholding of transform coefficients. Meanwhile, the external denoising method is good at recovering image details but may be largely distorted at the low frequency since the retrieved images have different illumination from the noisy image Therefore combining the internal and external denoising result, Pe and $\mathrm{P}^{\mathrm{i}}$ as follows

$$
\frac{\mathrm{P} 1^{s t}=T^{-1}\left(T_{2 D}\left(\mathrm{P}^{\mathrm{i}}\right) \odot \omega^{\mathrm{i}}+T_{2 D}\left(\mathrm{P}^{\mathrm{e}}\right) \odot \omega \mathrm{e}\right)}{{ }^{2} D}
$$

where $T_{2 D}$ and $T^{-1}{ }_{2 D}$ are forward and inverse 2D DCT transform ,respectively. The parameter $\odot$ is the point-wise multiplication. Matrix $\omega^{\mathrm{i}}$ and $\omega^{\mathrm{e}}$ are the weighting matrix to combine the frequency coefficients of Pi and Pe.

iii. Combined Image Denoising: The Second Stage a) Denoising by Wiener Filtering

Given one noisy patch $\mathrm{p}$ (in vector format), it is obtained through the observation model:

$$
\mathrm{p}=\mathrm{p}^{\mathrm{o}}+\mathrm{n}
$$

where $\mathrm{p}^{\mathrm{o}}$ is a noise free patch and $\mathrm{n}$ is white Gaussian noise with variance $\sigma^{2}$.They can be regarded as variables generated from stationary random processes, namely $\mathrm{p}^{\circ}$ and $\mathrm{n}$ are random vectors. The solution of this problem is a wiener filter :

$$
G=\mathbf{R}\left(\mathbf{R}+\sigma^{2} \mathbf{I}\right)^{-1}
$$

where the correlation matrix $\mathrm{R}=\mathrm{E}\{$ popo $T\}$ and $\mathrm{I}$ is the identify matrix. Since $\mathrm{R}$ is symmetric, it can be represented by eigenvalue decomposition as follows:

$$
\mathbf{R}=\mathbf{U}^{\wedge} \mathbf{U}^{\top}=\sum_{\substack{\boldsymbol{k} \\ \lambda_{k} \mathbf{u}_{k} \mathbf{u}^{\top} \boldsymbol{l}}}^{k}
$$

where $\mathrm{k}$ is the rank of $\mathrm{R}$. $\mathbf{u}_{k}$ is the eigenvector of $\mathrm{R}$, and $\lambda_{k}$ is the corresponding eigenvalue. $\mathrm{U}$ is the stacked eigenvectors $[\mathbf{u} 1, \mathbf{u} 2, \ldots, \mathbf{u} \boldsymbol{h}]$ and $\mathrm{A}$ is a diagonal matrix constructed by the eigenvalues.

\section{b) Denoising by Exploring External Correlation}

The noise in $\widetilde{I}^{1 s t}$ is greatly attenuated. Therefore, for each patch $\mathrm{P}^{1 s t}$ in $\sim^{1 s t}$,we can directly retrieve its $k_{2}$-NN patches $\left\{\mathrm{Q}_{1}, \mathrm{Q}_{2}, \ldots, \mathrm{Q}_{k 2}\right\}$ from the external image set $\left\{I^{r} 1, I^{r} 2\right.$, , $\left.I^{r K}\right\}$ using the baseline method. These patches $\left\{\mathrm{q}_{1}, \mathrm{q}_{2}, \ldots, \mathrm{q}_{k 2}\right.$
\} can be regarded as sample realizations of $\mathrm{p}^{\mathrm{o}}$. Therefore the matrix is estimated as:

\section{$k$}

$$
\begin{gathered}
\mathbf{R}=\underline{1} \sum \mathbf{q}_{i} \mathbf{q}^{\top} i \\
k 2 \quad i=1 \\
\mathbf{R}=\underline{1} \mathbf{q}^{2} \mathbf{D} \mathbf{q}^{\top} 2 D
\end{gathered}
$$

k2

where $\mathrm{q}_{2 D}=\left[\mathrm{q}_{1}, \mathrm{q}_{2}, \ldots, \mathrm{q}_{k 2}\right]$ is the matrix form of sample.

\section{CONCLUSIONS}

For any noisy image we first retrieve its correlated image set from web images instead of natural image. Then in the first stage external denoising part, a graph-cut patch matching to improve patch matching accuracy and in the internal denoising part similar noisy patch is filtered in the transform domain and then combining the internal and external denoising result and forward in the second stage in the second stage external denoising is done by using adaptive filtering in the noisy patches and internal denoising is done by using wiener filter on the noisy cube. By combining second stage external and internal denoising results, we obtain the final denoising result. For increasing the psnr value and quality of image we use wavelet filtering in internal denoising of second stage and wiener filtering in external denoising of second stage in our future work.

\section{ACKNOWLEDGMENT}

Our thanks to the teacher and my supporter who have contributed towards developments of the paper.

\section{REFERENCES}

[1]Kalpana, and Harjinder Singh,Nov.2015, "Review Paper:to study the image denoising techniques", Vol 02,pp.127-129

[2] Rohit Verma, and Dr. Jahid Ali,Oct.2013,"A Comparative study of various types of image noise and efficient noise removal techniques",vol.3, issue 10 , pp.617 -622.

[3] Priyanka Kamboj et al.,April.2013," Brief study of various noise model and filtering techniques", vol.4, No.4, pp.166-17.

[4] A. Buades, B. Coll, and J.-M. Morel,Jun.2005, “A nonlocal algorithm for image denoising," in Proc. IEEE Comput.So Conf. CVPR,pp. 60-65.

[5] D. Zoran and Y. Weiss,Nov,2011,"From learning models of natural image patches to whole image restoration," in Proc.IEEE ICCV, pp.479-486.

[6] I. Mosseri, M. Zontak, and M. Irani,Apr.2013 "Combining the power of internal and external denoising," in Proc.IEEE, pp. 1-9.

[7] H. Yue, X. Sun, J. Yang, and F. Wu,Jun.2014 "CID: Combined image denoising in spatial and frequency domains using web images," in Proc. IEEE Conf. CVPR,pp. 2933-2940.

[8] L. Dai, X. Sun, F. Wu, and N. Yu,Sep.2013 "Large scale image retrieval with visual groups," in Proc.20th IEEE Int.Conf.Image Process. (ICIP), pp. 2582-2586. 
[9] C. Barnes, E. Shechtman,2010,D. B. Goldman, and A.Finkelstein, "The generalized PatchMatch correspondence algorithm," in Proc. $11^{\text {th }}$ ECCV, pp. 2943.

[10] K. Dabov, A. Foi, V. Katkovnik, and K. Egiazarian,Aug.2007, "Image denoising by sparse 3-D transform-domain collaborative filtering," IEEE Trans.Image Process., vol. 16, no. 8, pp.2080-2095.
[11] H. C. Burger and S. Harmeling,2011, "Improving denoising algorithms via a multi-scale meta procedure," in Proc.33rd Int. Conf. Pattern Recognit., pp. 206-215.

[12] M. Zontak,I. Mosseri, and M. Irani,2013 "Separating signal from noise using patch recurrence across scales," in Proc. IEEE Conf. CVPR, pp. 1195-1202.

[13] M. Zontak and M. Irani,Jun.2011, "Internal statistics of a single natural image,"in Proc. IEEE Conf. CVPR, pp.977-984. 